\title{
Early experience with robotic mitral valve repair with intra-aortic occlusion
}

\author{
Risto Kesävuori, MD, Peter Raivio, MD, PhD, Janne J. Jokinen, MD, PhD, Antero Sahlman, MD,
}

Kari Teittinen, MD, and Antti Vento, MD, PhD

\section{ABSTRACT}

Objective: To report the learning curve and early results of robotic mitral valve repairs in comparison with propensity score-matched sternotomy controls after the adoption of a robotic mitral valve surgery program in a university teaching hospital.

Methods: A total of 142 patients underwent robotic mitral valve repair due to degenerative mitral regurgitation between May 2011 and December 2015. Control patients operated on via the conventional sternotomy approach were selected by the use of propensity score analysis resulting in 2 well-matched study groups.

Results: Valve repair rate was $98.6 \%$ and $97.9 \%$ in the robotic and sternotomy groups, respectively. Operation length, cardiopulmonary bypass, aortic crossclamp, and ventilation times were shorter in the sternotomy group. All of these times were statistically significantly reduced within the robotic group during the learning curve. Even though there was no statistically significant difference in the rate of perioperative complications between the groups, 3 patients in the robotic group required postoperative extracorporeal membrane oxygenation due to low cardiac output, and 1 patient in the robotic group died. In the robotic and sternotomy groups, $86.3 \%$ versus $84.7 \%$ of patients had grade $\leq 1+$ mitral valve regurgitation at the latest follow-up visit, and there was no statistically significant difference in survival or reoperation rate between the 2 study groups during follow-up.

Conclusions: The present series reports the entire early learning curve related to the introduction of robotic mitral valve repair in our institution. In all, repair rate and early durability were acceptable, but more patients in the robotic group had serious complications. Early major robotic complications that occurred may have been related to the simultaneous use of intra-aortic occlusion. (J Thorac Cardiovasc Surg 2018;155:1463-71)

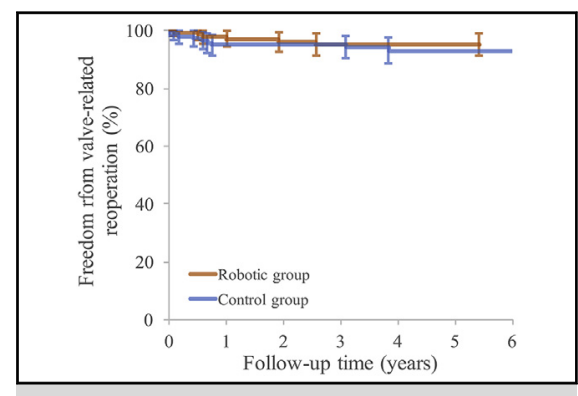

Freedom from valve-related reoperation.

\section{Central Message}

We report the early learning curve related to the introduction of robotic mitral valve repair Valve-related follow-up results were acceptable, but some major complications occurred in the robotic group. Early major robotic complications that occurred may have been related to the simultaneous use of intra-aortic occlusion.

\section{Perspective}

Excellent mid-term results after robotic mitra valve repair have been reported by high-volume centers. Our study reports the entire early learning curve related to the introduction of robotic mitral valve repair compared with propensity scorematched sternotomy controls. In all, repair rate and valve-related follow-up results were acceptable, but some major complications occurred in the robotic group. Early major robotic complications that occurred may have been related to the simultaneous use of intra-aortic occlusion.

See Editorial Commentary page 1472.

See Editorial page 1459

\footnotetext{
From the Department of Cardiac Surgery, Heart and Lung Center, Helsinki University Central Hospital, Helsinki, Finland.

This study was supported by grants from the Finnish Angiology Society.

Received for publication March 8, 2017; revisions received Sept 25, 2017; accepted

for publication Oct 9, 2017; available ahead of print Dec 6, 2017.

Address for reprints: Peter Raivio, MD, PhD, Helsinki University Central Hospital,

Heart and Lung Center, PO Box 340, Helsinki 00029, Finland (E-mail: peter.

raivio@hus.fi).

$0022-5223 / \$ 36.00$

Copyright (c) 2017 by The American Association for Thoracic Surgery

https://doi.org/10.1016/j.jtcvs.2017.10.076
}

Advancements in robotic surgical instrumentation and cardiopulmonary bypass technologies have expanded the role of robotically assisted operations in cardiac surgery.

$\square$ Scanning this QR code will take
you to a supplemental video for
this article.




\section{Abbreviations and Acronyms \\ $\mathrm{ECMO}=$ extracorporeal membrane oxygenator \\ $\mathrm{IQR}=$ interquartile range \\ MR = mitral valve regurgitation \\ $\mathrm{SD}=$ standard deviation}

Robotic methods are most widely adopted in mitral valve operations due to excellent visualization of the mitral valve using the robotic approach. In robotic mitral valve surgery, access to the heart is obtained by the use of small ports in the right intercostal spaces, allowing surgery to be performed with minimal tissue trauma in comparison with the conventional sternotomy approach. Suggested benefits of the robotically assisted approach have been faster return to ordinary daily activities, shorter length of stay, reduced pain, improved cosmesis, and reduced need for blood transfusions. In high-volume centers, the short-term and long-term results of robotic mitral valve surgery have been excellent, and outcomes have been comparable with the conventional sternotomy approach. ${ }^{1-8}$ Recently published expert opinion for practice guidelines of minimally invasive and robotic mitral valve surgery suggests that in the beginning of the learning curve only simple mitral valve pathologies should be operated via minimally invasive approaches. ${ }^{9-11}$ However, in high-volume centers, the results of complex robotic leaflet repairs have been comparable with more simple operations with no increase in the need of reoperations. ${ }^{7}$ Also, quality of life early after surgery improves faster after robotic operations when compared with the sternotomy approach, but this difference is reduced over time. ${ }^{12,13}$

The minimally invasive cardiac surgery program at the Helsinki University Central Hospital Heart and Lung Center was started in January 2009. The first minimally invasive operations were performed videothoracoscopically via a right mini-thoracotomy. Since May 2011, minimally invasive mitral valve operations have been performed with robotic assistance. In addition to robotic mitral valve surgery, robotically assisted coronary artery bypass grafting operations and robotically assisted operations for atrial septal defects and myxomas also have been performed at our institution.

The objective of this study was to report the learning curve after the adoption of a robotic mitral valve surgery program in a university teaching hospital and to report the early results of robotic mitral valve repair in comparison with propensity scorematched sternotomy controls. Notably, in our series the use of an endoaortic balloon was initiated at the same time with the robotic instrumentation, which resulted in overlapping learning curves. Also, myocardial protection methods evolved during the learning curve.

\section{PATIENTS AND METHODS \\ Patients and Data Collection}

A total of 145 consecutive patients underwent robotic mitral valve surgery at our institution between May 2011 and December 2015.
Operations were performed with the da Vinci Si Surgical System (Intuitive Surgical, Inc, Sunnyvale, Calif). Altogether, 3 patients who underwent a robotically assisted operation were excluded from this study, 2 due to a planned mitral valve replacement and 1 due to active endocarditis. This resulted in a study group of 142 patients who were scheduled for robotic mitral valve repair for degenerative mitral regurgitation.

Careful patient selection was carried out when selecting patients for robotic surgery. Exclusion criteria for robotic mitral valve surgery are listed in Table 1. Patients with significant comorbidities or high surgical risk were mostly operated on via the conventional sternotomy approach. To reduce selection bias, propensity score matching was used to create 2 study groups with similar preoperative risk profiles. ${ }^{14}$ The medical records of all patients who underwent isolated mitral valve surgery or mitral valve surgery with concomitant tricuspid valve annuloplasty or an atrial fibrillation ablation procedure from the conventional sternotomy approach in our institution between 2005 and 2015 were reviewed for the propensity score analysis. A total of 317 sternotomy patients were included in the analysis after we excluded patients who underwent urgent surgery, surgery for active endocarditis, or planned mitral valve replacement. Of these patients, a control group of 142 patients was selected using the propensity score analysis. Patients included in the study are depicted in a flow chart (Figure 1).

Preoperative patient characteristics of the robotic group and the propensity-matched control group are shown in Table 2. The majority of patients had isolated posterior leaflet pathology, but isolated anterior leaflet and bileaflet pathologies also were present in both study groups with no statistically significant difference between the 2 groups. All control patients and 141 patients in the robotic group had grade $3+$ or $4+$ mitral valve regurgitation (MR) preoperatively, and 1 patient in the robotic group who underwent concomitant myxoma excision had grade $2+$ MR preoperatively. A total of $19(13.4 \%)$ patients in the sternotomy group and $7(4.9 \%)$ patients in the robotic group had chronic lung disease preoperatively $(P=.022)$. Patients with chronic obstructive pulmonary disease were excluded from robotic surgery, which may in part explain the observed difference. Preoperatively, all patients underwent a computed tomography scan of the aorta and femoral vessels to exclude patients unsuitable for robotic surgery. Echocardiographic follow-up visits were performed mostly at 3 months and 1 year postoperatively, and all available data from the latest follow-up visits were included in this study.

The patient data were collected from intensive care, cardiac surgery, and cardiology databases retrospectively. Medical records related to preoperative and postoperative care were retrieved from other hospitals when needed. This study was approved by the local institutional board and the local ethics committee.

\section{Surgical Technique}

All robotically assisted operations were performed with the da Vinci Si Surgical system (Intuitive Surgical, Inc). Cardiopulmonary bypass was established by groin cannulation and aortic occlusion was performed primarily with an endoaortic balloon. A double-lumen endotracheal tube or a bronchial blocker was applied to allow isolated left-lung ventilation. The camera port was placed near the mammilla, mostly in the fourth intercostal space. The service port was placed laterally from the camera port to the same or adjacent intercostal space. The ports for the second, first, and third robotic arms were positioned using the 4-finger distance rule in the third, fifth, and sixth intercostal spaces, respectively.

Bicaval venous cannulation was performed via the right femoral and jugular veins with Medtronic, Inc (Minneapolis, Minn), Estech Systems Inc (Plano, Tex), or Edwards Lifesciences (Irvine, Calif) venous cannulas. Usually right-sided femoral arterial cannulation was preferred with a 21- or 23-F side branch arterial cannula (EndoReturn; Edwards Lifesciences). An endoaortic balloon (EndoClamp or IntraClude; Edwards Lifesciences) was positioned in the ascending aorta under echocardiographic control. The patient's right side was elevated $30^{\circ}$ from the horizontal plane, and the 
TABLE 1. Exclusion criteria for surgery

Significant aortic, iliac, or femoral artery calcification

Tiny femoral vessels

LV dysfunction

Pulmonary artery pressure $>70 \mathrm{~mm} \mathrm{Hg}$ or severe RV dysfunction

Diameter of the ascending aorta $>40 \mathrm{~mm}$

Significant mitral annular calcification

Moderate or severe aortic regurgitation

Kyphoscoliosis and pectus excavatum

Morbid obesity

Severe pleural adhesions (previous thoracic trauma, lung surgery, pleuritis)

Significant coronary stenosis

COPD

$\overline{L V}$, Left ventricle; $R V$, right ventricle; $C O P D$, chronic obstructive pulmonary disease.

da Vinci Si Surgical system was docked to the patient. After docking, cardiopulmonary bypass was started and a pericardiotomy was performed $4 \mathrm{~cm}$ anterior to the phrenic nerve. The pericardium was suspended with retraction sutures placed through angiocatheters through the thoracic wall.

Aortic occlusion was performed primarily with the endoaortic balloon, but the Chitwood clamp (Scanlan International, Inc, St Paul, Minn) also was used in some operations. Rapid right ventricular pacing or adenosine was used when positioning the endoaortic balloon. Myocardial protection methods were improved during the learning curve. Initially, the operations were performed solely with antegrade Bretschneider cold cardioplegia
(Custodiol HTK Solution; Essential Pharmaceuticals, LLC, Ewing, NJ) via the endoaortic balloon, but in the later operations antegrade cold blood cardioplegia was used and also retrograde cold blood cardioplegia was delivered via a ProPlege coronary sinus catheter (Edwards Lifesciences). The EndoClamp endoaortic balloon was replaced with the more recent IntraClude device, and the perfusion temperature was lowered to $32^{\circ} \mathrm{C}$ in the later operations.

Patients in the control group underwent mitral surgery via the conventional sternotomy approach. Cardiopulmonary bypass was established with direct cannulation of the ascending aorta, and a crossclamp was used for aortic occlusion. The heart was protected with cold ante- and retrograde blood cardioplegia. A short video of a robotic mitral valve operation performed at our institution is presented in Video 1.

\section{Statistical Analysis and Propensity Score Matching}

IBM SPSS, version 24.0 (IBM Corp, Armonk, NY) was used for statistical analysis. The Kolmogorov-Smirnov test was performed to test the normality of continuous variables. Continuous variables are reported as median and interquartile range (IQR) or mean and standard deviation (SD), and nominal values are reported as counts and percentages. Survival and freedom from valve-related reoperation was assessed with the Kaplan-Meier estimate. To compare differences between the 2 study groups, either the Mann-Whitney $U$ test or independent samples $t$ test for continuous variables, $\chi^{2}$ or Fisher exact test for categorical variables, and log-rank test for Kaplan-Meier estimates were used. The $\alpha$-level was set at 0.05 for statistical significance.

To reduce selection bias, a control group for patients in the robotic group was selected using propensity score matching. Propensity scores

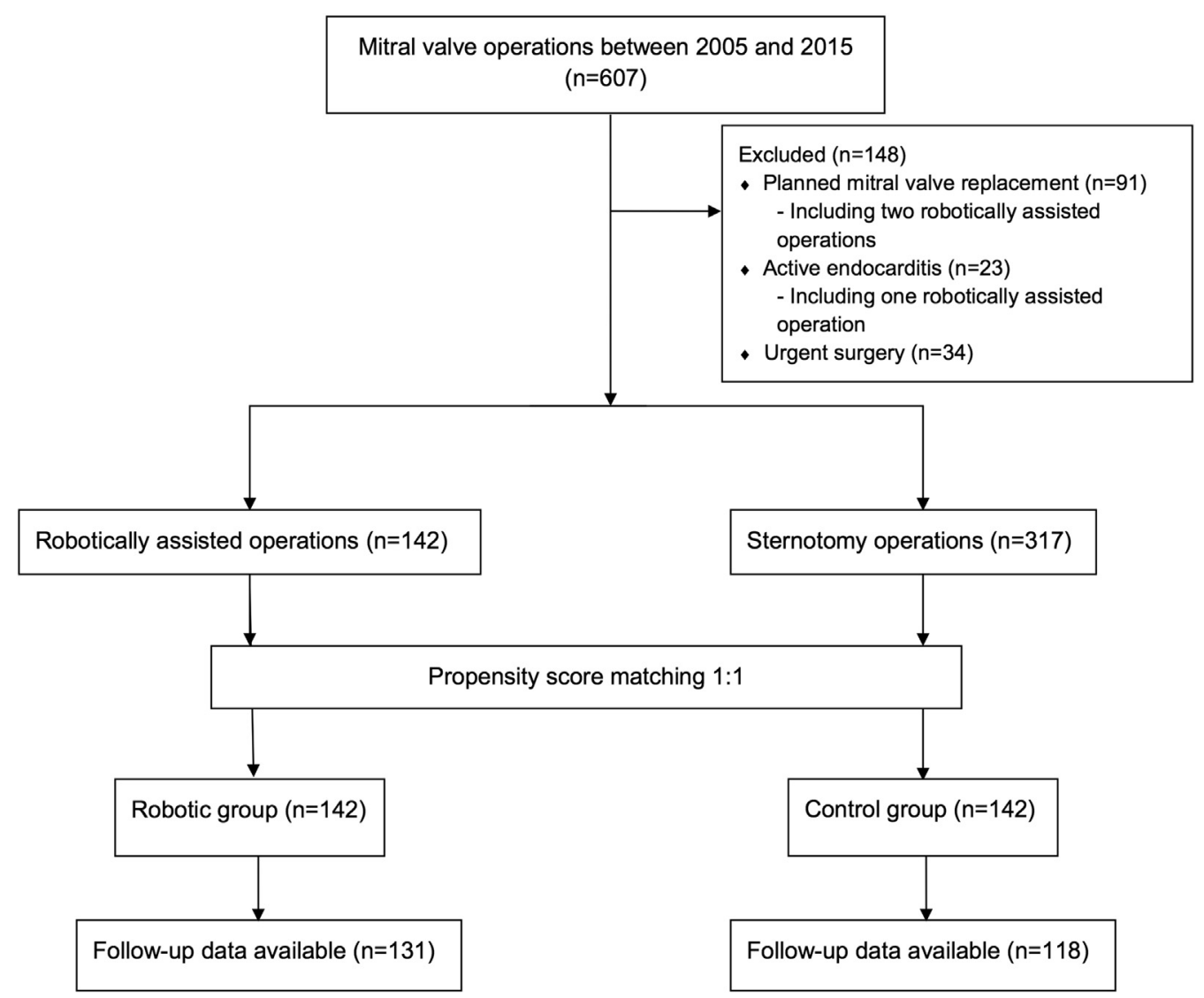

FIGURE 1. Patient flow diagram depicting mitral valve operations (isolated mitral valve operations and mitral valve operations with concomitant tricuspid valve annuloplasty or atrial fibrillation ablation procedure) between 2005 and 2015 . 
TABLE 2. Patient characteristics and risk factors

\begin{tabular}{|c|c|c|c|c|}
\hline Patient characteristics & Robotic group $(n=142)$ & Control group $(n=142)$ & $P$ value & Standardized difference \\
\hline \multicolumn{5}{|l|}{$\operatorname{Sex}^{*}$} \\
\hline Male & $115(80.9)$ & $113(79.5)$ & .882 & 0.04 \\
\hline Age, $y^{*}$ & $59.0(10.8)$ & $59.4(10.3)$ & .984 & 0.04 \\
\hline $\mathrm{BSA}, \mathrm{m}^{2 *}$ & $1.99(0.22)$ & $1.99(0.29)$ & .942 & 0.02 \\
\hline GFR, $\mathrm{mL} / \mathrm{min}^{*}$ & $89(76-110)$ & $88(72-111)$ & .899 & 0.02 \\
\hline Logistic EuroSCORE I, \%* & $2.08(1.51-3.09)$ & $2.23(1.51-3.19)$ & .378 & 0.01 \\
\hline Atrial fibrillation* & $47(33.1)$ & $46(32.4)$ & 1.000 & 0.02 \\
\hline Ejection fraction* & & & .832 & 0.05 \\
\hline$>50 \%$ & $129(90.8)$ & $131(92.3)$ & & \\
\hline$\leq 50 \%$ & $13(9.2)$ & $11(7.7)$ & & \\
\hline NYHA* & & & .741 & \\
\hline I & $18(12.7)$ & $13(9.2)$ & & 0.11 \\
\hline II & $63(44.4)$ & $61(43.0)$ & & 0.03 \\
\hline III & $56(39.4)$ & $63(44.4)$ & & 0.10 \\
\hline IV & $5(3.5)$ & $5(3.5)$ & & 0 \\
\hline Diabetes & $6(4.2)$ & $5(3.5)$ & 1.000 & \\
\hline Hypertension & $48(33.8)$ & $39(27.5)$ & .303 & \\
\hline Chronic lung disease & $7(4.9)$ & $19(13.4)$ & .022 & \\
\hline Myocardial infarction & $1(0.7)$ & 0 & 1.000 & \\
\hline Stroke & $1(0.7)$ & $1(0.7)$ & 1.000 & \\
\hline History of PCI & 0 & $1(0.7)$ & 1.000 & \\
\hline Previous cardiac surgery & 0 & 0 & 1.000 & \\
\hline MR & & & .518 & \\
\hline Grade $1+$ & 0 & 0 & & \\
\hline Grade $2+$ & $1(0.7)$ & 0 & & \\
\hline Grade $3+$ & $87(61.3)$ & $83(58.5)$ & & \\
\hline Grade 4+ & $54(38.0)$ & $59(41.5)$ & & \\
\hline Carpentier classification & & & 1.000 & \\
\hline I & $2(1.4)$ & $1(0.7)$ & & \\
\hline II & $140(98.6)$ & $141(99.3)$ & & \\
\hline Mitral pathology (Carpentier II) & & & .182 & \\
\hline Posterior leaflet & $120(84.5)$ & $109(76.8)$ & & \\
\hline Anterior leaflet & $11(7.7)$ & $16(11.3)$ & & \\
\hline Bileaflet & $9(6.3)$ & $16(11.3)$ & & \\
\hline LA diameter, mm & $49.9(7.1)$ & $51.7(7.8)$ & .141 & \\
\hline
\end{tabular}

Data shown as number of patients (\%) or mean \pm standard deviation (range) or median (interquartile range). BSA, Body surface area; GFR, glomerular filtration rate; EuroSCORE, European System for Cardiac Operative Risk Evaluation; NYHA, New York Heart Association; PCI, percutaneous coronary intervention; MR, mitral valve regurgitation; $L A$, left atrium. *Propensity-matched variables.

for each patient in the robotic group and for the 317 sternotomy patients were calculated using 8 preselected variables listed in Tables 2 and 3, including sex, age, body surface area, glomerular filtration rate, logistic EuroSCORE I, atrial fibrillation, ejection fraction, and New York Heart Association class. The resulting propensity scores were used to select control patients for the 142 patients in the robotic group in a $1: 1$ ratio using 0.02 match tolerance. Matching was performed with the nearestneighbor algorithm without replacement and resulted in a well-matched control group of 142 patients with regard to all 8 variables used in the propensity score matching. Standardized differences were calculated to assess the quality of the propensity score matching, and values $<0.1$ were considered a good match.

\section{RESULTS \\ Operative Data}

Operative data are presented in Table 4. In the robotic group, mitral valve repair was performed successfully in 140 operations of the 142 intended repairs, with a repair rate of $98.6 \%$. The remaining 2 patients received a biological prosthesis after an unsuccessful mitral repair. Correspondingly, in the sternotomy group 2 patients received a biological prosthesis, and 1 patient received a mechanical prosthesis after unsuccessful mitral repair 


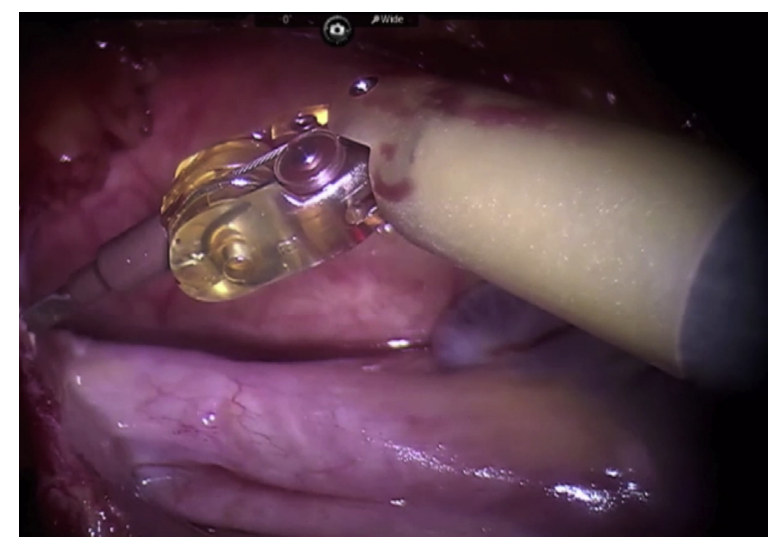

VIDEO 1. Robotic mitral valve repair. Video available at: http://www. jtcvsonline.org/article/S0022-5223(17)32410-8/fulltext.

with a repair rate of $97.9 \%$ for the sternotomy group. Table 4 presents the different mitral valve repair techniques used in the operations. In both study groups, mostly either neochord implantation or leaflet resection were performed to treat mitral regurgitation. Neochordae were implanted more frequently in the robotic group in comparison with the sternotomy group (78 [55.7\%] vs 54 [38.8\%]), whereas leaflet resection was performed more often in the sternotomy group (52 [37.1\%] vs 86 [61.9\%] in the robotic and sternotomy groups, respectively).

Concomitant surgery included $35(24.6 \%)$ versus 30 $(21.1 \%)$ atrial fibrillation ablation procedures, $14(9.9 \%)$ versus $7(4.9 \%)$ patent foramen ovale closures, and $6(4.2 \%)$ versus $17(12.0 \%)$ tricuspid valve annuloplasties in the robotic and sternotomy groups, respectively. There was one concomitant myxoma excision and one pericardial cyst excision in the robotic group. In the sternotomy group, one concomitant thymoma excision was performed.

The procedure-related times, ventilation, intensive care unit, and hospitalization times for both study groups are listed in Tables 4 and 5. Operation length, cardiopulmonary bypass time, aortic crossclamp time, and ventilation time were all statistically significantly shorter in the sternotomy group, whereas patients in the robotic group were transferred earlier from the intensive care unit to the ward. The total volume of chest tube drainage was similar between the study groups during the first postoperative 16 hours. There was no statistically significant difference in the number of patients requiring more than 1 unit of red blood cells during hospitalization between the study groups.

During the first 30 robotic operations, intraoperative times tended to shorten, and after the first 30 operations a steady state was reached. To describe the learning curve concerning robotically assisted operations, operationrelated times of the first 30 robotic operations and the last 112 robotic operations are presented in Table 6. Operation length, console time, cardiopulmonary bypass time, crossclamp time, and ventilation time were all statistically significantly reduced during the learning curve.

\section{Conversions and Complications}

In all, 13 robotically assisted operations were converted to sternotomy, and 1 operation was converted to thoracotomy with a conversion rate of $9.9 \%$. As our series includes the early part of the learning curve, the threshold of

TABLE 3. Baseline patient characteristics before propensity score matching

\begin{tabular}{|c|c|c|c|c|}
\hline Patient characteristics & Robotic group $(n=142)$ & Prematched control $(n=317)$ & $P$ value & Standardized difference \\
\hline \multicolumn{5}{|l|}{ Sex } \\
\hline Male & $115(80.9)$ & $223(70.3)$ & .016 & 0.25 \\
\hline Age, y & $59.0(10.8)$ & $62.4(11.4)$ & .001 & 0.30 \\
\hline $\mathrm{BSA}, \mathrm{m}^{2}$ & $1.99(0.22)$ & $1.94(0.25)$ & .032 & 0.19 \\
\hline GFR, $\mathrm{mL} / \mathrm{min}$ & $83(72-93)$ & $79(65-91)$ & .009 & 0.24 \\
\hline Logistic EuroSCORE I, \% & $2.08(1.51-3.09)$ & $2.23(1.51-3.19)$ & $<.001$ & 0.33 \\
\hline Atrial fibrillation & $47(33.1)$ & $144(45.4)$ & .010 & 0.25 \\
\hline Ejection fraction & & & .171 & 0.16 \\
\hline$>50 \%$ & $129(90.8)$ & $272(85.8)$ & & \\
\hline$\leq 50 \%$ & $13(9.2)$ & $45(14.2)$ & & \\
\hline NYHA & & & .051 & \\
\hline I & $18(12.7)$ & $26(8.2)$ & & 0.14 \\
\hline II & $63(44.4)$ & $128(40.4)$ & & 0.08 \\
\hline III & $56(39.4)$ & $131(41.3)$ & & 0.04 \\
\hline IV & $5(3.5)$ & $32(10.1)$ & & 0.26 \\
\hline
\end{tabular}

Data shown as number of patients $(\%)$ or mean \pm standard deviation (range) or median (interquartile range). BSA, Body surface area; GFR, glomerular filtration rate; EuroSCORE, European System for Cardiac Operative Risk Evaluation; NYHA, New York Heart Association. 
TABLE 4. Operative data

\begin{tabular}{|c|c|c|c|}
\hline Variable & Robotic group $(n=142)$ & Control group $(n=142)$ & $P$ value \\
\hline Operation length, min & $254(227-290)$ & $217(173-251)$ & $<.001$ \\
\hline Console time, $\min$ & $129(112-154)$ & - & \\
\hline CPB time, $\min$ & $157(135-181)$ & $112(93-154)$ & $<.001$ \\
\hline Crossclamp time, $\min$ & $104(87-120)$ & $86(67-109)$ & $<.001$ \\
\hline $\begin{array}{l}\text { Mitral procedure } \\
\text { Repair } \\
\text { Bioprosthesis } \\
\text { Mechanical prosthesis }\end{array}$ & $\begin{array}{c}140(98.6) \\
2(1.4) \\
0\end{array}$ & $\begin{array}{c}139(97.9) \\
2(1.4) \\
1(0.7)\end{array}$ & .605 \\
\hline \multicolumn{4}{|l|}{ Concomitant surgery } \\
\hline AF ablation & $35(24.6)$ & $30(21.1)$ & .480 \\
\hline PVI & 0 & $5(3.5)$ & .060 \\
\hline Left atrial maze & $35(24.6)$ & $23(16.2)$ & .105 \\
\hline Biatrial maze & 0 & $4(2.8)$ & .123 \\
\hline Tricuspid repair & $6(4.2)$ & $17(12.0)$ & .028 \\
\hline PFO closure & $14(9.9)$ & $7(4.9)$ & .172 \\
\hline LAA ligation & $32(22.5)$ & $26(18.3)$ & .392 \\
\hline Myxoma excision & $1(0.7)$ & 0 & 1.000 \\
\hline Thymoma excision & 0 & $1(0.7)$ & 1.000 \\
\hline Pericardial cyst excision & $1(0.7)$ & 0 & 1.000 \\
\hline Mitral valve repair technique & $(\mathrm{n}=140)$ & $(\mathrm{n}=139)$ & \\
\hline Neochord implantation & $78(55.7)$ & $54(38.8)$ & .004 \\
\hline Anterior leaflet & $16(11.4)$ & $29(20.9)$ & .032 \\
\hline Posterior leaflet & $65(46.4)$ & $30(21.6)$ & $<.001$ \\
\hline Annuloplasty & 139 (99.3) & $139(100)$ & 1.000 \\
\hline Leaflet resection & $52(37.1)$ & $86(61.9)$ & $<.001$ \\
\hline Commissuroplasty & $18(12.9)$ & $19(13.7)$ & .860 \\
\hline Cleft closure & $42(30.0)$ & $37(26.7)$ & .530 \\
\hline Leaflet plication & $5(3.6)$ & $5(3.6)$ & 1.000 \\
\hline Edge to edge & 0 & $1(0.7)$ & 1.000 \\
\hline
\end{tabular}

Data shown as number of patients (\%) or mean \pm standard deviation (range) or median (interquartile range). $C P B$, Cardiopulmonary bypass; $A F$, atrial fibrillation; $P V I$, pulmonary vein isolation; $P F O$, patent foramen ovale; $L A A$, left atrial appendage.

conversion to full sternotomy was kept low to maximize the safety of the procedure. Five conversions were due to problems with endoclamp positioning and cardioplegia delivery. Four operations were converted after suboptimal mitral valve repair. In 2 of these operations, a mitral valve replacement was performed and 2 valves were re-repaired via sternotomy. There were 2 conversions for bleeding, 1 conversion due to pleural adhesions, 1 due to problems with venous return, and 1 because of robotic malfunction.

A total of 11 patients in the robotic group and 8 patients in the sternotomy group required reoperations due to bleeding (Table 5). Eight patients in the robotic group were reoperated due to pleural hematoma. Two patients who underwent conversion to sternotomy were reoperated due to retrosternal bleeding during hospitalization. One patient in the robotic group required resternotomy after pericardial effusion and cardiac tamponade. In the sternotomy group, 5 patients underwent resternotomy due to retrosternal bleeding, and 1 patient required thoracotomy for pleural hematoma. Two patients in the robotic group and 6 patients in the sternotomy group required subxiphoidal fenestrations due to pericardial effusion postoperatively.

In the robotic group, low cardiac output and iatrogenic injuries were more common than in the open group ( $7 / 142$ vs $1 / 142$, respectively, $P=.066$, Table 5 ). In the robotic group, a total of 3 patients required extracorporeal membrane oxygenator (ECMO) support postoperatively due to low cardiac output (Table 5). One of these patients died during hospitalization due to multiorgan failure after 2 reoperations due to bleeding and after implantation of a left ventricular assist device. The other 2 patients requiring ECMO support were discharged after prolonged hospitalization (16 days and 31 days) with no MR. In 2 robotically assisted operations, the left circumflex coronary artery was occluded during annuloplasty. One of these patients was treated with percutaneous coronary intervention and a coronary stent placement, and the other required a coronary artery bypass grafting operation after an unsuccessful percutaneous coronary intervention. In 3 robotic operations, an intra-aortic balloon pump was applied intraoperatively including the 2 patients requiring ECMO support. One 
TABLE 5. Results

\begin{tabular}{|c|c|c|c|}
\hline Variable & $\begin{array}{c}\text { Robotic } \\
\text { group } \\
(n=142)\end{array}$ & $\begin{array}{c}\text { Control } \\
\text { group } \\
(n=142)\end{array}$ & $\begin{array}{c}P \\
\text { value }\end{array}$ \\
\hline ICU stay, d & $1(1-1)$ & $1(1-2)$ & .011 \\
\hline Ventilation time, $\mathrm{h}$ & $15(12-20)$ & $13(10-17)$ & .001 \\
\hline Hospitalization time, $\mathrm{d}$ & $7(6-8)$ & $7(6-9)$ & .190 \\
\hline Chest tube $16-\mathrm{h}$ drainage, $\mathrm{mL}$ & $580(420-825)$ & $550(415-800)$ & .396 \\
\hline $\begin{array}{l}\text { Red blood cell requirement } \\
\quad \geq 2 \text { units }\end{array}$ & $41(28.9)$ & $38(26.8)$ & .746 \\
\hline Second pump run & $9(6.3)$ & $8(5.6)$ & 1.000 \\
\hline $\begin{array}{l}\text { Conversions } \\
\text { Sternotomy } \\
\text { Thoracotomy } \\
\text { Endoclamp-related } \\
\text { Residual MR } \\
\text { Bleeding } \\
\text { Other }\end{array}$ & $\begin{array}{r}14(9.9) \\
13(9.2) \\
1(0.7) \\
5(3.5) \\
4(2.8) \\
2(1.4) \\
3(2.1)\end{array}$ & & \\
\hline \multicolumn{4}{|l|}{ Complications } \\
\hline 30-d mortality & $1(0.7)$ & 0 & 1.000 \\
\hline Perioperative stroke & 0 & $1(0.7)$ & 1.000 \\
\hline Perioperative MI & $1(0.7)$ & $3(2.1)$ & .622 \\
\hline Low cardiac output syndrome & $4(2.8)$ & $1(0.7)$ & .371 \\
\hline IABP implantation & $3(2.1)$ & $1(0.7)$ & .622 \\
\hline Sepsis & $2(1.4)$ & 0 & .498 \\
\hline Empyema & $1(0.7)$ & 0 & 1.000 \\
\hline Pneumonia & $5(3.5)$ & 0 & .060 \\
\hline Wound infection & $2(1.4)$ & $1(0.7)$ & 1.000 \\
\hline Dialysis & $3(2.1)$ & $3(2.1)$ & 1.000 \\
\hline LCx occlusion & $2(1.4)$ & 0 & .498 \\
\hline Femoral artery occlusion & $1(0.7)$ & 0 & 1.000 \\
\hline \multicolumn{4}{|l|}{ Reoperations } \\
\hline Due to bleeding & $11(7.7)$ & $8(5.6)$ & .636 \\
\hline ECMO implantation & $3(2.1)$ & 0 & .247 \\
\hline PM implantation & $1(0.7)$ & $2(1.4)$ & 1.000 \\
\hline LCx occlusion & $2(1.4)$ & 0 & .498 \\
\hline Cross-over bypass & $1(0.7)$ & 0 & 1.000 \\
\hline
\end{tabular}

Data shown as number of patients $(\%)$ or mean \pm standard deviation (range) or median (interquartile range). ICU, Intensive care unit; $M R$, mitral valve regurgitation; $M I$, myocardial infarction; $I A B P$, intra-aortic balloon pump; $L C x$, left circumflex coronary artery; $E C M O$, extracorporeal membrane oxygenator; $P M$, pacemaker.

patient in the sternotomy group required an intra-aortic balloon pump postoperatively. One patient in the robotic group required crossover bypass after arterial occlusion of the right femoral artery. Two patients in the sternotomy group and 1 patient in the robotic group underwent a pacemaker implantation due to atrioventricular block.

Infectious complications were more frequent in the robotic group ( $P=.066$, Table 5$)$. In the robotic group, altogether 7 patients had postoperative infectious complications including 2 superficial wound infections, 5 cases of pneumonia, 1 catheter-related sepsis, and 1 empyema and sepsis. In the sternotomy group, 1 patient had a superficial wound infection. Three patients in the robotic group and 2 patients in the sternotomy group required dialysis due to renal insufficiency postoperatively. A total of 1 and 3 patients had perioperative myocardial infarction in the robotic and sternotomy groups, respectively. There were no cerebrovascular events in the robotic group, but 1 patient in the sternotomy group had a computed tomography-confirmed stroke perioperatively.

\section{Follow-up and Valve-Related Reoperations}

Follow-up results are presented in Table 7. Patients in the robotic surgery group who were converted to open sternotomy or thoracotomy were included in the follow-up data of the robotic group. At the latest echocardiographic follow-up visit (median 15 [IQR 3-23] months vs 21 [IQR 7-57] months), $86.3 \%$ versus $84.7 \%$ of patients had grade $1+$ or less MR in the robotic and sternotomy groups, respectively. Follow-up data were available from $92.3 \%$ versus $83.1 \%$ patients in the robotic and sternotomy groups, respectively. Six $(4.1 \%)$ patients in the robotic group and $10(7.0 \%)$ patients in the sternotomy group required reoperations due to residual MR during follow-up (mean 35 [SD 17] months vs 64 [SD 35] months, for the robotic and sternotomy groups, respectively). Because of the difference in lengths of the follow-up periods, survival and freedom from reoperation were evaluated with the Kaplan-Meier estimate. Figures 2 and 3 illustrate Kaplan-Meier curves for survival and freedom from valve-related reoperation, respectively. During follow-up, there was no statistically significant difference in survival or in valve-related reoperation rate between the 2 study groups $(P=.656$ and $P=.841$, respectively).

\section{COMMENT}

This study presents the results and early learning curve of robotic mitral valve repair operations in comparison with propensity-matched controls. The resulting propensitymatched control group and the robotic group were similar regarding the 8 variables used in the propensity score matching. Repair rate was high in both study groups, but the repair techniques differed between the study groups. Neochordae were implanted more commonly in the robotic group, whereas leaflet resection was performed more often in the sternotomy group. The conversion rate was high, and more than one third of the conversions were endoclamp-related. The procedure-related times and ventilation times were shorter in the sternotomy group, but a statistically significant reduction of these times was observed in the robotic group during the learning curve. Even though there was no statistically significant difference in the overall rate of perioperative complications between the robotic and sternotomy groups, in the robotic group, 3 patients required postoperative ECMO support, 2 patients suffered from left circumflex coronary artery occlusion, and 1 patient died. 
TABLE 6. Learning curve

\begin{tabular}{|c|c|c|c|}
\hline Variable & $\begin{array}{c}\text { Robotic group } \\
5 / 2011 \text { to } 5 / 2012(n=30)\end{array}$ & $\begin{array}{c}\text { Robotic group } \\
6 / 2012 \text { to } 12 / 2015(n=112)\end{array}$ & $P$ value \\
\hline Operation length, min & 277 (247-349) & $250(224-281)$ & .002 \\
\hline Console time, $\min$ & $150(124-215)$ & $122(110-147)$ & $<.001$ \\
\hline $\mathrm{CPB}$ time, $\min$ & $171(148-199)$ & $151(133-175)$ & .002 \\
\hline Crossclamp time, min & $111(95-138)$ & $101(86-116)$ & .045 \\
\hline Ventilation time, $\mathrm{h}$ & $20(14-24)$ & $14(12-17)$ & .001 \\
\hline ICU time, $\mathrm{d}$ & $1(1-2)$ & $1(1-1)$ & .070 \\
\hline Hospitalization time, $\mathrm{d}$ & $7(6-8)$ & $7(6-8)$ & .907 \\
\hline Chest tube $16-\mathrm{h}$ drainage, $\mathrm{mL}$ & $620(475-1035)$ & $573(413-818)$ & .238 \\
\hline
\end{tabular}

Data shown as median (interquartile range). $C P B$, Cardiopulmonary bypass; $I C U$, intensive care unit.

During follow-up, survival and freedom from valve-related reoperation were similar between the 2 study groups.

Previously, similar complication and reoperation rates have been reported in robotic and sternotomy mitral valve repair operations. ${ }^{1,2,5}$ In addition, excellent mid-term results after robotic mitral valve repair have been reported by highvolume centers. ${ }^{2-5,7}$ In this study, we aimed to report all complications in detail. Even though the difference in the overall rate of complications was statistically not significant, the robotic technique was associated with some major complications that may be related to both the learning curve and to a different approach in the robotic group with regard to both cardioplegia and repair techniques. Our study presents the early learning curve related to the introduction of robotic mitral valve repair, which explains the reduction of intraoperative times during the learning curve within the robotic group. However, despite taking this reduction into consideration, the operative and ventilation times observed in this study are longer than those reported by high-volume centers. ${ }^{2-5,7}$

Unexpectedly, 3 patients in the present series required postoperative ECMO support due to low cardiac output. This underlines the importance of myocardial protection. To improve myocardial protection, in our series singledose Bretschneider cold cardioplegia was replaced with intermittent cold antegrade and retrograde blood cardioplegia and the perfusion temperature was lowered.

The greatest limitation of this study is its retrospective design. The patients scheduled for robotic mitral valve

TABLE 7. Latest MR status during follow-up

\begin{tabular}{lccr}
\hline $\begin{array}{c}\text { Mitral valve } \\
\text { regurgitation }\end{array}$ & $\begin{array}{c}\text { Robotic group } \\
(\mathbf{n}=\mathbf{1 3 1})\end{array}$ & $\begin{array}{c}\text { Control group } \\
(\mathbf{n}=\mathbf{1 1 8})\end{array}$ & $\boldsymbol{P}$ value \\
\hline Grade 1+ or less & $113(86.3)$ & $100(84.7)$ & .857 \\
Grade 2+ & $5(3.8)$ & $4(3.4)$ & 1.000 \\
Grade 3+ & $5(3.8)$ & $2(1.7)$ & .451 \\
Grade 4+ & $2(1.5)$ & $1(0.8)$ & 1.000 \\
Reoperated & $7(5.3)$ & $10(8.5)$ & .619 \\
\hline
\end{tabular}

Data shown as number of patients (\%). repair were selected. Even though propensity score matching resulted in well-matched pairs of study patients and controls, it does not eliminate the effect of inherent intention-to-treat or selection bias. The 8 variables that were used to calculate the propensity score may not include all relevant risk factors affecting the outcome of mitral valve surgery. Mitral valve anatomy and the presence of concomitant operations were not included in the propensity score, which is a limitation. Also, it was not possible to match the groups based on operative variables such as cardioplegia delivery and aortic occlusion strategy. There might also be risk factors that remain unrecognized and are therefore missing from our data. An important limitation of this study is that the mitral valve repair techniques were significantly different between the groups. In the robotic group, more nonresection techniques were used. Also, cardioplegia and aortic occlusion strategies were different in the 2 groups. In addition, fewer concomitant tricuspid operations were performed in the robotic group. Patients in the control group were also more historical in comparison with the patients in the robotic group because of the smaller number of sternotomy

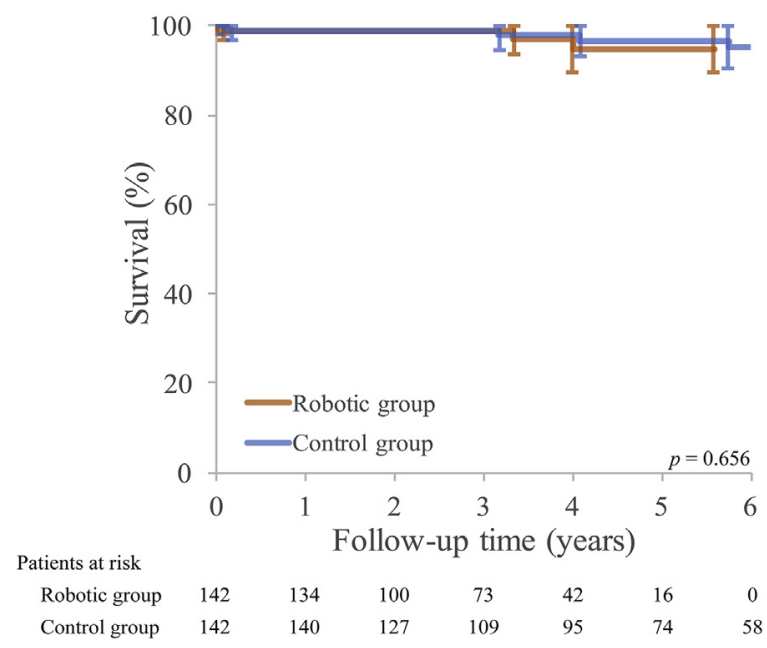

FIGURE 2. Survival. 


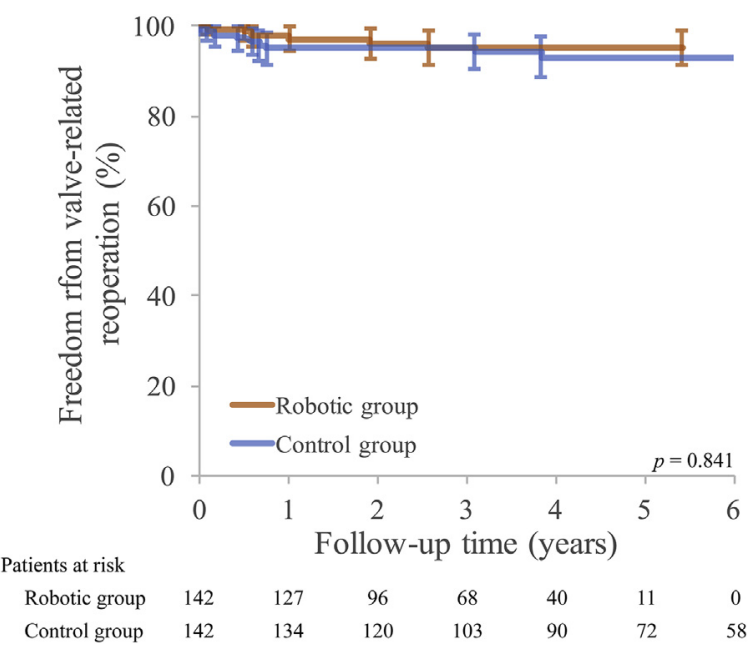

FIGURE 3. Freedom from valve-related reoperation.

mitral operations after the introduction of robotic mitral surgery at our institution. To take into account the resulting differences in the lengths of the follow-up periods, Kaplan-Meier estimates for survival and freedom from valve-related reoperations were constructed with no statistically significant differences between the study groups. Data from the latest echocardiographic follow-up visits were available from $92.3 \%$ versus $83.1 \%$ patients in the robotic and sternotomy groups, respectively. Because of the partly missing follow-up data, the number of patients at risk is reduced in the Kaplan-Meier analyses. This leads to a significant reduction of statistical power with increasing follow-up time.

The safety of robotic mitral valve repair was inferior to that of open repair in this series. In our experience, we initiated robotic instrumentation and the use of an endoartic balloon simultaneously, which resulted in overlapping learning curves of these 2 new technologies. Also, myocardial protection methods evolved during our experience. We recommend that similar institutions with a limited annual count of mitral valve operations would start their robotic mitral valve program with a dedicated and constant surgical team. Only one console surgeon with an underlying intensive training period might be preferable in the beginning of the learning curve. We also recommend that there are dedicated perfusionist, anesthesiologist, and scrub nurse teams involved. Also, we suggest that the use of an endoaortic balloon should not be started at the same time as a robotic surgery program is initiated due to overlapping learning curves. An anesthesiologist with comprehensive skills with the transesophageal ultrasound is required in the operating room not only during the placement of the endoaortic balloon but also during the whole surgical procedure. Every effort should be made to detect endoballoon migration and to ensure adequate cardioplegia delivery, adequate drainage, and adequate cooling. Finally, very careful patient selection is warranted.

The present series reports the entire early learning curve related to the introduction of robotic mitral valve repair with intra-aortic occlusion in our institution. In all, repair rate and valve-related follow-up results were acceptable, but some major complications occurred in the robotic group.

\section{Conflict of Interest Statement}

Drs Vento and Sahlman are Proctor of Intuitive Surgical 2014. All other authors have nothing to disclose with regard to commercial support.

\section{References}

1. Cao C, Wolfenden H, Liou K, Pathan F, Gupta S, Nienaber TA, et al. A metaanalysis of robotic vs. conventional mitral valve surgery. Ann Cardiothorac Surg. 2015;4:305-14

2. Mihaljevic T, Jarrett CM, Gillinov AM, Williams SJ, DeVilliers PA, Stewart WJ, et al. Robotic repair of posterior mitral valve prolapse versus conventional approaches: potential realized. J Thorac Cardiovasc Surg. 2011;141:72-80.e1-4.

3. Murphy DA, Moss E, Binongo J, Miller JS, Macheers SK, Sarin EL, et al. The expanding role of endoscopic robotics in mitral valve surgery: 1,257 consecutive procedures. Ann Thorac Surg. 2015;100:1675-81; discussion 1681-2.

4. Nifong LW, Rodriguez E, Chitwood WR Jr. 540 consecutive robotic mitral valve repairs including concomitant atrial fibrillation cryoablation. Ann Thorac Surg. 2012;94:38-43.

5. Suri RM, Burkhart HM, Daly RC, Dearani JA, Park SJ, Sundt TM III, et al Robotic mitral valve repair for all prolapse subsets using techniques identical to open valvuloplasty: establishing the benchmark against which percutaneous interventions should be judged. J Thorac Cardiovasc Surg. 2011 142:970-9.

6. Suri RM, Dearani JA, Mihaljevic T, Chitwood WR Jr, Murphy DA, Trento A, et al. Mitral valve repair using robotic technology: safe, effective, and durable. J Thorac Cardiovasc Surg. 2016;151:1450-4.

7. Suri RM, Taggarse A, Burkhart HM, Daly RC, Mauermann W, Nishimura RA, et al. Robotic mitral valve repair for simple and complex degenerative disease: midterm clinical and echocardiographic quality outcomes. Circulation. 2015; 132:1961-8

8. Yoo JS, Kim JB, Jung SH, Kim DH, Choo SJ, Chung CH, et al. Mitral durability after robotic mitral valve repair: analysis of 200 consecutive mitral regurgitation repairs. J Thorac Cardiovasc Surg. 2014;148:2773-9.

9. Ailawadi G, Agnihotri AK, Mehall JR, Wolfe JA, Hummel BW, Fayers TM, et al. Minimally invasive mitral valve surgery I: patient selection, evaluation, and planning. Innovations (Phila). 2016;11:243-50.

10. Wolfe JA, Malaisrie SC, Farivar RS, Khan JH, Hargrove WC, Moront MG, et al Minimally invasive mitral valve surgery II: surgical technique and postoperative management. Innovations (Phila). 2016;11:251-9.

11. Lehr EJ, Guy TS, Smith RL, Grossi EA, Shemin RJ, Rodriguez E, et al. Minimally invasive mitral valve surgery III: training and robotic-assisted approaches. Innovations (Phila). 2016;11:260-7.

12. Suri RM, Antiel RM, Burkhart HM, Huebner M, Li Z, Eton DT, et al. Quality of life after early mitral valve repair using conventional and robotic approaches. Ann Thorac Surg. 2012;93:761-9.

13. Yang M, Yao M, Wang G, Xiao C, Wu Y, Zhang H, et al. Comparison of postoperative quality of life for patients who undergo atrial myxoma excision with robotically assisted versus conventional surgery. J Thorac Cardiovasc Surg. 2015;150:152-7.

14. Rubin DB. The design versus the analysis of observational studies for causa effects: parallels with the design of randomized trials. Stat Med. 2007;26:20-36.

Key Words: minimally invasive surgical procedures, robotic surgical procedures, cardiac surgical procedures, mitral valve insufficiency, adult 